\title{
Im System verstrickte Zivilgesellschaft
}

\section{Sean O'Siochrù and Bruce Girard}

\section{(2) OpenEdition \\ 1 Journals}

Electronic version

URL: http://journals.openedition.org/sjep/568

DOI: 10.4000/sjep.568

ISSN: 1663-9677

Publisher

Institut de hautes études internationales et du développement

\section{Printed version}

Date of publication: 1 novembre 2003

Number of pages: $215-227$

ISSN: $1660-5926$

\section{Electronic reference}

Sean O'Siochrù und Bruce Girard, «Im System verstrickte Zivilgesellschaft », Schweizerisches Jahrbuch für Entwicklungspolitik [Online], 22-2 | 2003, Online erschienen am: 10 Juni 2010, abgerufen am 08 September 2020. URL : http://journals.openedition.org/sjep/568 ; DOI : https://doi.org/10.4000/sjep. 568 


\section{Im System verstrickte Zivilgesellschaft}

\section{Sean 0'Siochrù* und Bruce Girard**}

D

er Weltgipfel über die Informationsgesellschaft (WSIS) wurde mit aufrichtigen Hoffnungen auf eine neue Art Gipfeltreffen der Vereinten Nationen in die Wege geleitet. In UNO- und Regierungskreisen setzte sich die Auffassung durch, dass das konventionelle Gipfelmodell ausgedient hatte. Einige argumentierten, die aufeinander folgenden Veranstaltungen hätten zu „Gipfelmüdigkeit“ geführt. Der Ausdruck „Gipfeltreffen“ weckt Bilder von Delegierten in dunklen Anzügen, die sich von einer langweiligen Sitzung in die nächste schleppen; doch der Begriff umfasst eine weit komplexere und nuanciertere Wirklichkeit: Dazu gehören Tendenzen zum Unilateralismus, insbesondere in den USA, das wachsende Gewicht von Nicht-UN-Organisationen wie z. B. die Welthandelsorganisation (WTO) und der G-8, die damit verbundenen Schwierigkeiten, im UNO-Kontext signifikante Fortschritte zu erzielen, das offensichtliche Fehlen echter Folgemassnahmen nach den Gipfeltreffen und sogar absichtliche Versuche, die UNO zu umgehen. Wie die „Gebermüdigkeit“ verbirgt der Begriff „Gipfeltreffen“ mehr Motive, als er enthüllt.

Auch die Organisationen der Zivilgesellschaft äussern zunehmend Skepsis an den konventionellen Gipfeln. Zu oft wurden ihre Anliegen von den offiziellen Treffen ausgegrenzt und sie selbst in Paralleltreffen mit dürftigem Zugang zur Hauptveranstaltung abgeschoben. Während der Weltfrauenkonferenz von Beijing im Jahr 1995 wurde das Treffen der 35'000 Delegierten der Nichtregierungsorganisation über 50 Kilometer vom offiziellen Tagungsort entfernt ausgerichtet.

Deshalb werden Aussichten auf eine neue Art Gipfeltreffen in etlichen Kreisen begrüsst. Als sich jedoch die Internationale Fernmeldeunion (ITU) für den WSIS stark machte, kannte sie diesen Hintergrund wohl nur schlecht, da sie dem Kern des UNO-Systems und auch den zahlreichen Gipfeln nie wirklich nahe gekommen war ${ }^{1}$. Die ITU definierte sich selbst immer als Fachorganisation, und die dünnere Luft am Gipfel bedeutete eine neue Erfahrung. Früh wurde geargwöhnt, die ITU verspreche deshalb einen innovativen Ansatz für WSIS, weil sie den konventionellen Ansatz mit allen möglichen Fallstricken gar nicht kenne. Das hat sich diesmal bewahrheitet, obwohl bei anderen Unternehmungen Unschuld nicht immer nachteilig sein muss.

* Schriftsteller, Aktivist, internationaler Konsulent im Kommunikations- und Medienbereich, Irland.

** Journalist und Forscher im Bereich Mediengouvernanz, Niederlande.

1 Es wurde nie überzeugend erklärt, weshalb nicht die UNESCO oder gar das UNDP die federführende Organisation für den Gipfel sein sollte. Das UNDP zeigte kein Interesse, die UNESCO aber sehr wohl und stand sogar im Begriff, einen Gipfel nach eigenen ähnlichen Vorstellungen zu prüfen. Beide Organisationen haben mit den inhaltlichen Themen der Informationsgesellschaft mehr zu tun als die ITU, die sich hauptsächlich um die Aspekte der Infrastruktur kümmert. 
In der Folge soll der Kontext der Beteiligung der Zivilgesellschaft an UNO-Gipfeln beschrieben werden. Anschliessend wird die Frage erörtert, ob der WSIS so innovativ war bzw. ist, wie behauptet wurde. Ausserdem sollen die der Situation zu Grunde liegenden Ursachen geprüft und kurz die Perspektiven für den WSIS betrachtet werden.

\section{Hintergrund}

Nichtregierungsorganisationen (NRO) haben in der UNO und an den Weltgipfeln von Anfang an eine aktive Rolle gespielt. Bereits im Jahr 1948 erhielten die ersten NRO einen Beratungsstatus im Wirtschafts- und Sozialrat der Vereinten Nationen (ECOSOC). Die ersten Regeln betreffend diese Interaktionen wurden vom ECOSOC im Jahr 1950 verabschiedet ${ }^{2}$ und $1968^{3}$ sowie $1996^{4}$ überarbeitet. Damit unterliegen die Beziehungen der UNO, ihrer Mitgliedsgruppen und Versammlungen zu den NRO einem klaren Katalog von Regeln, die seither mehrmals - das letzte Mal in der Millenniums-Erklärung mit der Erneuerung des Mandats - bestätigt, erklärt und verfeinert wurden ${ }^{5}$.

Die Regeln beschreiben die Zusammensetzung der NRO: Es handelt sich um internationale Organisationen, die nicht von einer Regierungsstelle oder durch eine zwischenstaatliche Vereinbarung eingesetzt werden. Daneben wird auf nationale, subregionale und regionale NRO Bezug genommen. Gewinnorientierte Vereinigungen werden selbstverständlich ausgeklammert, z.B. mit der Bedingung, dass Ziel und Zweck der Organisationen mit dem Geist, den Zielen und den Grundsätzen der Charta der Vereinten Nationen übereinstimmen müssten. Allerdings können Unternehmen über ihre Industrieverbände vertreten sein. Obwohl die NRO nicht explizit von anderen Organisationen der Zivilgesellschaft getrennt werden, sehen die Regeln zur Akkreditierung bei einzelnen UNO-Stellen (z.B. Organisation der Vereinten Nationen für Bildung, Wissenschaft und Kultur UNESCO, Entwicklungsprogramm der Vereinten Nationen UNDP) oder bei Gipfeln in der Praxis durchaus Unterschiede vor. Auflagen wie ein fester Sitz, eine demokratisch verabschiedete Verfassung und Jahresberichte tendieren dazu, weite Teile der Zivilgesellschaft formell auszuschliessen.

Die genaue Auslegung und Durchsetzung der Regeln ist die Aufgabe der jeweiligen Gipfel und beruht letztlich auf der Einigung der Regierungen, die mit einigen Variationen auch stets zu Stande gekommen ist. Es wurden Wege gefunden, um andere Akteure einzubeziehen, z.B. mit den Multi-Stakeholder-Dialogsitzungen, die erstmals in der Tagung der UNCED (Konferenz der Vereinten Nationen über Umwelt und Entwicklung) im Jahr 1992 eingeführt und später vom Weltgipfel über nachhaltige Entwicklung (WSSD) ${ }^{6}$ übernommen wurden. Auch die Idee der „dreigliedrigen Partizipation“ steht mittlerweile hoch im Kurs. Diese Entwicklung hängt zusammen mit dem Aufkommen der „öffent-

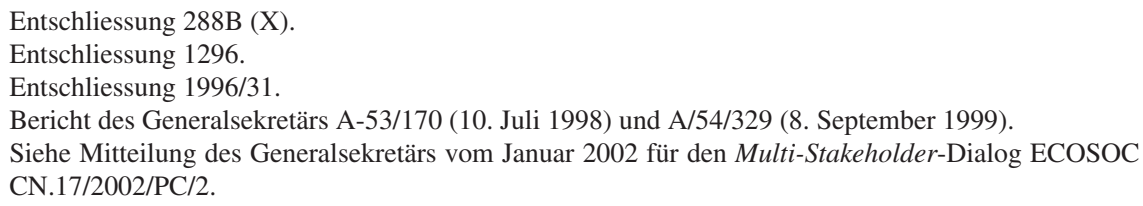


lich-privaten Partnerschaften“ - bisweilen mit einer symbolischen Beteiligung der NRO - als bevorzugter Entwicklungsträger der Regierungen, die besonders in ärmeren Ländern handels- und marktorientierte Politiken durchsetzen. Häufig handelt es sich aber um eine beschönigende Beschreibung für die Privatisierung und um das Eingeständnis, dass der Privatsektor - unterstützt von der Weltbank und verschiedenen regionalen Pendants - mittlerweile den Löwenanteil der Investitionsressourcen kontrolliert.

\section{Die KMU bleiben ausgeschlossen}

Der Weltgipfel über die Informationsgesellschaft verfolgt die Ambition, die vier Komponenten Regierungen, internationale Organisationen, Zivilgesellschaft und Privatsektor auf intensive und innovative Weise zusammen zu führen. Die Organisatoren wussten sehr wohl, dass die beiden letzten Komponenten besonderer Motivierung bedurften; deshalb schlugen sie ihnen ein bekömmliches und attraktives Menü vor und erlaubten ihnen eine wirkliche Teilnahme. In dieser Hinsicht war es zwingend notwendig, dass der Gipfel nicht nur die grossen multinationalen Konzerne, sondern über die lokalen nationalen und internationalen Verbände und Fachvereinigungen auch die kleinen und mittleren Unternehmen mit einbezog.

Die kleinen und mittleren Unternehmen (KMU) bilden in der Tat das Rückgrat des Privatsektors. Der Erfolg des Gipfels steht und fällt mit der Einbeziehung der KMU. Zwar bieten sich die grossen multinationalen Unternehmen als natürliche Partner an, was aufwändige Infrastrukturen und Technologien anbelangt (Zugang und Hardware). Aber die KMU des IKT-Sektors sind Garanten der Inhalte mit sämtlichen Folgeerscheinungen: Transfer von Know-how und Software, vielfältige originelle und lokale Inhalte, Anwendungen in der E-Governance und im E-Commerce, Talentsuche, Bildung von Humanressourcen von hoher Qualität, Fragen des geistigen Eigentums und der Piraterie.

Die KMU und ihre Verbände wie z.B. der Internationale Verband der Multimedia-Vereinigungen (Fédération Internationale des Associations du Multimédia FIAM) und auf schweizerischer Ebene Swissmedia tun sich jedoch schwer, ihren Platz in der Architektur des Gipfels zu finden. In der Schweiz wurden sie beispielsweise nicht einmal eingeladen, an der von der Bundesverwaltung eingerichteten dreigliedrigen Plattform teilzunehmen, welche auf die Entwürfe der Erklärung und des Aktionsplans reagieren soll - als ob mit der Einladung der Internationalen Handelskammer (IHK) und economiesuisse von einer Vertretung des gesamten Privatsektor ausgegangen werden könnte.

Diese beiden Lobby-Organisationen haben zwar die Aufgabe, die KMU zu vertreten; aber das ist kein Grund, um Verbände auszuschliessen, welche die KMU ebenfalls bzw. noch spezifischer repräsentieren. Ein Ausschluss wäre gravierend, denn er hätte zwei Auswirkungen: Zum einen müsste auf alle Beiträge der KMU verzichtet werden (Rückmeldungen von der Arbeit vor Ort, an der Basis gelebte Erfahrungen, praktische Kenntnisse und dynamische Kompetenzen), zum anderen würde die grundlegende und kulturelle Vielfalt der zahllosen Nichtregierungsorganisationen (NRO), welche die Zivilgesellschaft bilden, ausser Acht gelassen.

Aus diesem Grund ist zweierlei unbedingt erforderlich: Erstens muss entschieden bzw. eingestanden werden, dass die Zivilgesellschaft sich nicht auf kapital-, globalisierungs- und profitfeindliche NRO beschränkt, sondern auch die NRO des Privatsektors umfasst. Zweitens müssen diese NRO voll in den Gipfelprozess integriert werden, um neben den multinationalen Unternehmen, den Mitgliedern der Internationalen Fernmeldeunion (ITU) und der IHK die Interessen der KMU zu vertreten.

Angesichts der aktuellen Entwicklung stellt sich allen Ernstes die Frage, ob die generell halbherzigen Bestrebungen, die KMU und ihre Verbände einzubeziehen, nicht auf ein politisches Doppelmanöver hindeuten: Zum einen soll ein bestimmtes ideologisches Bild der Zivilgesellschaft aufrechterhalten werden und zum anderen sollen die Privilegien der Grossunternehmen, die Mitglied eines Sektors der Internationalen Fernmeldeunion sind, verteidigt werden.

* Von Roland Grunder, Generalsekretär von SwissMedia - Schweizer Verband Multimedia. 
Ausmass und Art der förmlichen Beteiligung der NRO und der übrigen Akteure sollten nicht überbewertet werden. Nur die Regierungen haben das Recht, Vorschläge einzureichen, oder die Befugnis, in den eigentlichen Gipfeltreffen abzustimmen. In der Regel sind die Gelegenheiten, Unterlagen zu unterbreiten und vor der Versammlung das Wort zu ergreifen, begrenzt und klar umrissen. Geändert haben sich die informellen Mittel der Einflussnahme auf den Prozess. Der Privatsektor verfügte stets über eine starke Lobby-Maschinerie, die normalerweise hinter verschlossenen Türen von mächtigen Ländern kontrolliert wurde. Erst seit kurzem melden sich die NRO innerhalb und ausserhalb der Gipfel in unterschiedlichem Ausmass zu Wort. Bis 2001 wurde starker und nachhaltiger Druck ausgeübt, um die Türen auch formell weiter zu öffnen. Mit der Machtübernahme der Bush-Regierung in den USA, ihrer unternehmensfreundlichen Agenda und ihrer feindlichen Einstellung gegenüber Multilateralismus und jeglicher Opposition hat sich jedoch seither das allgemeine Umfeld erneut verändert - inwieweit, bleibt noch umfassend zu prüfen.

Zur Komplexität trägt ausserdem bei, dass der UNO-Generalsekretär unlängst ein „Panel von hochrangigen Persönlichkeiten“ beauftragt hat, das Verhältnis zur Zivilgesellschaft zu überprüfen und die Zivilgesellschaft breiter, d.h. über die gängige Definition der NRO hinaus, zu definieren und einzubeziehen ${ }^{7}$.

Das Bild der Partizipation steht demnach noch im Wandel. Zwar sind einige Fixpunkte zu erkennen, aber die internen Kräfte zielen in unterschiedliche Richtungen und drohen selbst die Fixpunkte mitzureissen. Was kann folglich der WSIS an Innovation anbieten?

\section{Der WSIS und der dreigliedrige Ansatz}

Trotz der konzeptuellen Unklarheit beruhte der innovative Anspruch des WSIS von Anfang an auf dem Ziel, als erste hochrangige Gipfelveranstaltung die Regierungen, die Zivilgesellschaft und den Privatsektor in einem einschliessenden dreigliedrigen Ansatz zu umfassen ${ }^{8}$. Der hohe ITU-Beamte, welcher im Dezember 2002 in Barcelona der Global Community Networking-Konferenz den WSIS-Gipfel vorstellte, unterstrich die Bedeutung der Beteiligung der Zivilgesellschaft und erklärte, die umfassende Beteiligung der Zivilgesellschaft in der Vorbereitung des Gipfels werde von der ITU, den Vereinten Nationen und von UNO-Generalsekretär Kofi Annan vorbehaltlos unterstützt. Was unter „umfassender Beteiligung“ zu verstehen ist, wurde nicht ausbuchstabiert, aber Mitglieder des Sekretariats und auch die ITU sehen darin zweifellos eine Chance, über die Oppositionspolitik hinauszugehen und einen stärker konsensgestützten Ansatz zu finden, den sie für ein wesentliches Merkmal der Informationsgesellschaft halten.

7 Strengthening of the United Nations: An agenda for further change. UNO-Generalversammlung A/57/387. 9. September 2002.

8 Die Gliederung des WSIS-Gipfels in zwei Teile wird bisweilen auch als Neuerung behandelt, was potenziell auch zutrifft; allerdings wird nicht behauptet, dass diese Neuerung geplant wurde; man geht weithin davon aus, dass es sich um einen Kompromiss handelt, weil die beiden betreffenden Länder den Gipfel ausrichten wollten. 
Der innovative Anspruch hat sich rasch zu einem Markenzeichen der Rhetorik über den WSIS entwickelt. Er wurde in Unterlagen und Erklärungen der WSISFührung, darunter des Generalsekretärs der ITU, des Vorsitzenden des Vorbereitungsprozesse und des Exekutivdirektors des WSIS-Sekretariats, wiederholt.

Diese Tatsache zeitigte folgende Konsequenzen:

1) Erstens sollte das Sekretariat drei getrennte Abteilungen für die einzelnen Stakeholder-Gruppen umfassen.

2) Zweitens sollten die Zivilgesellschaft und der Privatsektor vollständig in den Gipfel einbezogen werden - vom Vorbereitungsprozess bis zu den Schlussergebnissen. Das hätte in der Tat ein Novum bedeutet; deshalb war verständlich, dass in der Frühphase des Prozesses nur wenig Detailinformationen vorlagen.

Die Abteilung Zivilgesellschaft (Civil Society Division, CSD) wurde als erste im WSIS-Sekretariat gegründet und bezog Ende 2001 die frisch renovierten Räumlichkeiten am ITU-Sitz. Die innovative dreigliedrige Struktur des Sekretariats geriet jedoch rasch in Schwierigkeiten. Die Regierungs- bzw. zwischenstaatliche Abteilung (governmental/intergovernmental division) hatte einen schweren Start und ist personell unterbesetzt. Der Privatsektor ging auf Distanz zum Sekretariat und zog es vor, über die Internationale Handelskammer zu arbeiten und das Sekretariatsbüro für den Privatsektor leer stehen zu lassen.

Das Potenzial für Innovationen wurde des weiteren durch die unerklärlichen Verzögerungen bei der Einstellung des Exekutivdirektors des Sekretariats weiter geschwächt; das Amt wurde Ende 2000 ausgeschrieben, aber der Exekutivdirektor wurde erst anlässlich der Tagung des ITU-Rates im April 2002 - einige Monate vor der ersten PrepCom - ernannt.

\section{Anfängliche Hoffnungen und die Rolle der UNESCO}

Ein dreigliedriges Sekretariat sollte nur als Mittel zum Zweck dienen; grundsätzlich konnte die Erneuerung, den Prozess und die Ergebnisse betreffend, fortgesetzt werden. Die CSD bewies Beharrlichkeit und engagierte sich energisch für die Einbeziehung der Zivilgesellschaft. Sie förderte die Bemühungen einer unabhängigen Arbeitsgruppe über die Beteiligung der Zivilgesellschaft, welche innovative Vorschläge für die tatsächliche Beteiligung der Zivilgesellschaft im WSIS-Prozess ausarbeiten und diese durch die Schlüsselveranstaltungen bis und mit der PrepCom begleiten sollte' .

Im Februar 2002 hielt die UNESCO mit den NRO und mit Organisationen der Zivilgesellschaft vier Konsultationsrunden ab. Der Schwerpunkt lag auf thematischen Bereichen und auf der Formulierung von Empfehlungen, die letztlich in die Erklärung und den Aktionsplan des WSIS einfliessen sollten. Nachdem die Vorschläge für den Inhalt des WSIS vorlagen, stellte sich die dringende Frage,

9 Die Arbeitsgruppe erstellte zwei Berichte, Modalities for Civil Society Participation in the WSIS: Issues and Options und Civil Society Participation in the WSIS: Issues and Principles. Die Berichte wurden anlässlich der fünften Konsultationssitzung der UNESCO in Paris im April 2002 unterbreitet und können auf folgender Website eingesehen werden: <http://comunex.comunica.org/wsis/docs.htm.>. 
wie die Vorschläge in den Prozess eingebettet werden sollten. Die UNESCO und die CSD richteten mit vereinten Kräften eine fünfte Konsultationsrunde aus, um die Vorschläge zu überprüfen und - noch wichtiger - die Modalitäten der Beteiligung der Zivilgesellschaft am WSIS zu erörtern und zu planen. Die Tagung verabschiedete eine Erklärung, in welcher eine Reihe von Leitgrundsätzen für die Beteiligung der Zivilgesellschaft umrissen und verschiedene zu ergreifende Massnahmen vorgeschlagen werden. Die Teilnehmer forderten einen transparenten WSIS-Vorbereitungsprozess und verlangten, „dass die Akteure der Zivilgesellschaft in inhaltlichen Ausführungen zur Tagesordnung gleich behandelt werden sollten wie die Nationalstaaten und die Unternehmen des Privatsektors“. Ausserdem verpflichteten sie sich, zu gewährleisten, dass die Beteiligung der Zivilgesellschaft ,ausgewogen ist, und dass die Stimme aller gehört wird, wobei das geografische Gleichgewicht und die Gleichstellung der Geschlechter zu berücksichtigen sind“. Im Schlussgrundsatz wird ein wesentliches Anliegen für die Zukunft des WSIS und besonders für die zweiteilige Gliederung des Gipfels niedergelegt: Die Zivilgesellschaft muss die Gelegenheit erhalten, gleichberechtigt mit den Regierungen und dem Privatsektor umfassend an der Überwachung und Umsetzung der Ergebnisse des Gipfels teilzunehmen und mitzuwirken ${ }^{10}$.

Die Konsultationsvorschläge der UNESCO bedeuteten die Krönung der Aspirationen der Zivilgesellschaft ${ }^{11}$ und umfassten folgende Punkte:

$\checkmark$ Es muss umgehend ein Prozess zur Förderung der Interaktion und Information über den WSIS gestartet werden, der vom Sekretariat und anderen Gebern unterstützt und ausserdem von existierenden Organisationen der Zivilgesellschaft geplant und durchgeführt wird. Die Mittel sollten neben dem Internet auch die traditionellen Medien (Radio, Printmedien usw.) sowie andere Kommunikationssysteme und Praktiken umfassen.

$\checkmark$ Ein Protokoll für die Informationsverbreitung und für die Transparenz im Zusammenhang mit dem WSIS sollte spezifisch vereinbart und getestet werden.

- Die Zivilgesellschaft sollte im Büro des Gipfels vertreten sein, um die Beteiligung am laufenden Vorbereitungsprozess zu gewährleisten und die Transparenz zu steigern.

$\square$ Die Beteiligung der NRO am Gipfel muss hinsichtlich ihrer Auswahl und der Einladung zum Weltgipfel präzisiert werden. Insbesondere müssen die Kriterien für die Beteiligung der NRO eindeutig festgelegt werden, um den Unterschied zwischen Lobby-Organisationen und NRO zu klären.

- Es sollte ein Fonds zur Unterstützung der effektiven und ausgewogenen Vertretung der Zivilgesellschaft eingerichtet werden. Die Staaten und Geberorganisationen sollten aufgefordert werden, Beiträge an den Fonds zu leisten, welche anschliessend nach Kriterien, die eine kompetente Stelle (z.B. der United Nations Non-Governmental Liaison Service UN NGLS) bestimmt, zugewiesen würden.

10 Der Beitrag der UNESCO an die PrepCom 1 des WSIS kann auf folgender Website eingesehen werden : <http://portal.unesco.org/ci/ev.php?URL_ID=2294\&URL_DO=DO_TOPIC\&URL_SECTION= 201\&reload=1047371556>.

11 Die Regionaltagung von Bamako veröffentlichte ausserdem eine deutliche Erklärung zur Beteiligung. 
$\square$ Die Dezentralisierung des Konsultations- und Mobilisierungsprozesses könnte durch regionale „Moderatoren“ innerhalb der bestehenden NRONetze gestärkt und mit angemessenen Ressourcen ausgestattet werden.

$\checkmark$ Die von der Zivilgesellschaft durchgeführten Konsultationen müssen offiziell anerkannt werden. Ausserdem sollten Mechanismen für die Einbindung der Ergebnisse in den offiziellen Prozess vorgesehen werden.

- Ein laufender Mechanismus für den Überwachungsprozess quer durch alle Bereiche der Informationsgesellschaft würde dazu beitragen, aktuelle und neue Anliegen zu erkennen.

$\square$ Der Gipfel sollte von einem NRO-Forum begleitet werden, in welchem die Zivilgesellschaft ihren Beitrag an den Prozess und die Ergebnisse erörtern kann.

Einige dieser Punkte gingen über die bisherigen Errungenschaften der Zivilgesellschaft hinaus (z.B. das Vertretungsbüro), und andere (z.B. das explizite Transparenz-Protokoll) übertrafen die etablierte Praxis. Im Grossen und Ganzen jedoch sind diese Vorschläge vor einem generell positiven Hintergrund weder als kostspielig noch als exzessiv zu beurteilen. Sie sind im Gegenteil im Ton durchaus gemässigt ausgefallen und streben lediglich allmähliche Fortschritte an.

\section{PrepCom1}

Alle Hoffnungen und Pläne wurden in einem prozeduralen Vakuum geschmiedet. Die PrepCom 1 (Juli 2002) bedeutete die erste Feuerprobe für den Konsens der Regierungen, die sich prompt ein hitziges Gefecht lieferten. Zahlreiche Regierungen - manche suchten Rückendeckung bei den wenigen, die unverhohlen ihre Meinung äusserten - stemmten sich kategorisch gegen die geringfügigste Abweichung von den eng ausgelegten förmlichen Verfahren der Regierungssouveränität über den Entscheidungsfindungsprozess. Versuche, neue Beteiligungsformen einzuführen, wurden durchweg abgeschmettert oder noch im Entwurfsstadium aufgegeben. Die abschliessende Plenarversammlung der Zivilgesellschaft anlässlich der PrepCom 1 kam zu folgendem Fazit: Nach der optimistischsten Interpretation bedeutet die Einigung zu den Regeln und Modalitäten für die Beteiligung vielleicht eine Abweichung von etablierten Praktiken, aber wenig positive Innovation, wie sie z.B. in der Konsultationsreihe der UNESCO mit Organisationen der Zivilgesellschaft und des Privatsektors anlässlich der Regionalkonferenz von Bamako beobachtet wurde ${ }^{12}$.

Zwar gab es anlässlich der PrepCom Ansätze von Erneuerung, aber aus der Perspektive der Zivilgesellschaft zielten sie in die falsche Richtung. Die PrepCom einigte sich auf ein praktisch beispielloses Zugeständnis betreffend die Beteiligung des Privatsektors in Form der Akkreditierung von individuellen Privatunternehmen. Die Plenarversammlung der Zivilgesellschaft zog dazu folgendes Fazit: Der Beschluss, individuelle kommerzielle Akteure in dieser Weise ohne angemessene Diskussion und ohne Verweise auf die etablierten Verfahren und Praktiken an

12 Unterstrichen im englischen Original. 
einem UNO-Gipfel zu beteiligen, ist beispiellos und wird sich auf das gesamte UNO-System auswirken. Unsere Sorge gilt nicht nur der Öffnung für die Beteiligung einer neuen Gruppe, sondern der Tatsache, dass erstens die geeigneten Diskussionen, Verhandlungen und Referenzen auf die Regelungen und Praktiken ausgeblieben sind, und dass zweitens die explizite Einschliessung von direkt profitorientierten Unternehmen der geltenden UNO-Regelung zuwider läuft. ${ }^{13}$

Mehrere Faktoren trugen zu diesem Ergebnis bei.

Zunächst wusste die ITU wenig über den ECOSOC und seine Regelungen. Die ITU widersetzte sich jahrelang und trotz der erheblichen konstruktiven Bemühungen der NRO selbst dem Drängen des ECOSOC, die NRO in ihrer Struktur anzuerkennen ${ }^{14}$. Die Ernennung eines ehemaligen ITU-Beamten an die Spitze des WSIS-Sekretariats war mit Blick auf die Einbindung des WSIS in die breiteren Erfahrungen mit Weltgipfeln ebenfalls ein unglücklicher Schachzug.

Zweitens weist die ITU innerhalb der UNO eine einzigartige Struktur auf: Unternehmen des Privatsektors können „Mitglieder“ der ITU werden; ihnen fehlt lediglich das Stimmrecht in Belangen der Statuten und der Übereinkommen der ITU. Das wurde im Gipfel übernommen - illegitimerweise insofern, als damit gegen die bestehenden ECOSOC-Regelungen verstossen wird.

Drittens herrschte generell der Eindruck, dass der private Sektor die Triebkraft der Informationsgesellschaft bildet und so voll einbezogen werden muss.

Im Ergebnis eine missliche Situation: Trotz des Ziels, den dreigliedrigen Ansatz zu fördern, wurde unter dem Strich der private Sektor bevorzugt. Dieser erhielt die traditionelle Akkreditierung über die Industrieverbände, deren Gewicht mit der Akkreditierung individueller Unternehmen noch zunahm. Überdies bestand die Möglichkeit - welche die Industrieverbände entsprechend nutzten -, im Lager der Zivilgesellschaft mitzumachen. So übernahmen Vereinigungen kommerzieller Verleger und andere, welche die Interessen der Industrie verfolgen, in den Beratungen der Zivilgesellschaft eine aktive Rolle. Die entwicklungsorientierte, nicht-kommerzielle Zivilgesellschaft, die verglichen mit dem Privatsektor und den Regierungen bereits mit einem enormen Ungleichgewicht an verfügbaren Ressourcen konfrontiert war, musste zudem die verzerrenden Auswirkungen der Einmischung des Privatsektors in ihren Bemühungen um schlüssige Positionen bekämpfen und sich als effektiver Partner im Prozess organisieren.

Kurz: Die PrepCom 1 erwies sich als Enttäuschung für diejenigen, die Erneuerung und eine stärkere Beteiligung der Zivilgesellschaft anstrebten. Der Grund zum Weitermachen wurde nachträglich weithin in Frage gestellt - weitergemacht wird lediglich in der Hoffnung, dass die Dinge sich zum Besseren wenden.

\section{Wende zum Besseren?}

13. Unterstrichen im englischen Original.

14 Eine ITU-Arbeitsgruppe mit Vertretern der NRO wurde eingesetzt, um diese und verwandte Fragen zu prüfen und im September 1999 Bericht zu erstatten. Allen Versprechen zum Trotz wurden die Empfehlungen ignoriert. Siehe <http://www.comunica.org/itu_ngo/.>. 


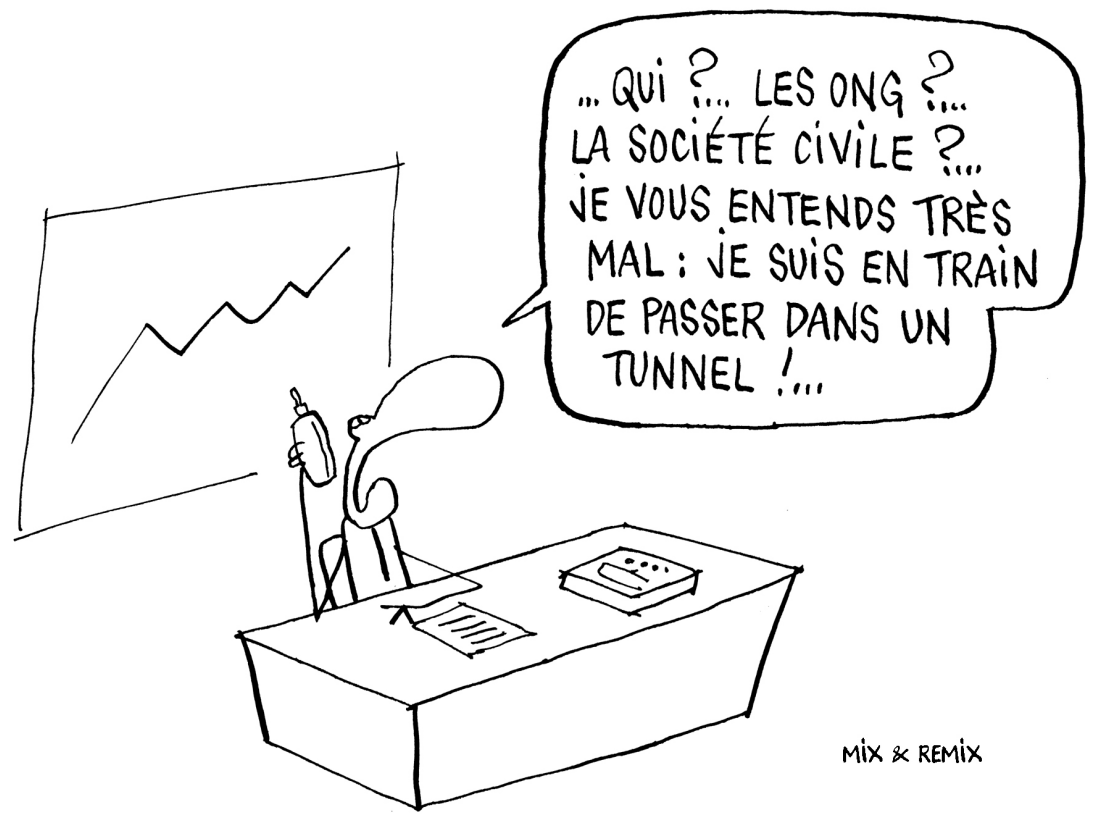

Wer? Die NGO ? Die Zivilgesellschaft?...

Ich höre Sie schlecht, ich stecke im Tunnel...

Die regionalen Vorbereitungskonferenzen boten einigen Anlass zur Hoffnung und bewiesen die Bereitschaft vieler bzw. wohl der meisten Länder, die Türen etwas weiter zu öffnen. In Bamako (vor der PrepCom 1), Bukarest, Tokio und Bávaro wurden zahlreiche Anliegen der Zivilgesellschaft zum Inhalt formell oder informell aufgenommen. Allerdings fehlte jegliche Rechtsgrundlage, so dass die Situation keinen Präzedenzfall für die PrepCom 2 begründete.

\section{PrepCom 2}

Die PrepCom 2 (Februar 2003) begann mit einer Reihe von Rundtischgesprächen der Multi-Stakeholder, an denen die - zwar willkürlich ausgewählte und nur am Rande an der Organisation beteiligte - Zivilgesellschaft relativ gut vertreten war. Allerdings wirkten sich die Ergebnisse der Rundtischgespräche, die von der zwischenstaatlichen Plenarversammlung zusammengefasst wurden, offensichtlich nur begrenzt auf den Entwurf der Erklärung bzw. des Aktionsplanes aus.

Inzwischen widmeten sich die Regierungen erneut der anspruchsvollen Aufgabe, sich - tagelang - über das Verfahren und hauptsächlich über die Frage der Beteiligung der Nichtregierungsorganisation zu streiten. Sogar der private 
Sektor verabschiedete sich aus dem Geschehen, so dass zur Abschlusssitzung nur noch wenige wackere Streiter übrig blieben. In der zweiten Woche forderte die Zivilgesellschaft von den Regierungen, sämtliche Sitzungen für Beobachter zu öffnen und formelle Foren für die Interaktion zwischen der Zivilgesellschaft und den wenigen Ländern der (geschlossenen) Redaktionsausschüsse zu schaffen. In diesem Bereich wurden einige Fortschritte erzielt, und es wurden formelle und informelle Mechanismen für die Zivilgesellschaft geschaffen, damit diese ihre Reaktionen auf die Entwürfe der Regierungen rechtzeitig und sichtbar äussern konnte. Allerdings beharrten einige Regierungen bis zum bitteren Ende auf ihren Positionen und spielten so lange auf Zeit, dass der Prozess beinahe blockiert wurde.

Das Endergebnis ist ein Abbild der Unentschlossenheit des zwischenstaatlichen Gremiums hinsichtlich Inhalt und Verfahren, einschliesslich der Beteiligung der Zivilgesellschaft. Wegen des unvollständigen Resultats musste in der Zeit vor der PrepCom 3 noch vieles geleistet werden, und obwohl die Zivilgesellschaft sich mit der impliziten Anerkennung ihrer Positionen und Vorschlägen trösten konnte, fehlte erneut eine klare Verpflichtung für die Beteiligung der Zivilgesellschaft während bzw. zwischen den Tagungen. Es zeigte sich, dass jegliche Zugeständnisse bei zahlreichen Ländern auf unvermindert hartnäckigen Widerstand stossen würden.

Trotzdem begann die Zivilgesellschaft mit dem Aufbau ihrer eigenen Organisation. Das Büro der Zivilgesellschaft wurde von der Plenarversammlung der Zivilgesellschaft anlässlich der PrepCom 2 im Anschluss an eine erste Tagung mit rund zwei Dutzend ausgewählten Vertretern, die im Januar 2003 von der CSD einberufen worden war, formell eingesetzt. Das Büro der Zivilgesellschaft befasst sich nicht mit Inhaltsfragen (den substanziellen Punkten, die am WSIS angesprochen werden), sondern mit dem Prozess. Ziel ist es, einen Mechanismus zur Erleichterung der Beziehungen mit der zwischenstaatlichen Seite (einschliesslich des WSIS-Büros), dem privaten Sektor und anderen zu schaffen und gleichzeitig die Akteure der Zivilgesellschaft zu befähigen, ihre Tätigkeiten kohärent zu organisieren und neue Akteure aufzunehmen. Die Zivilgesellschaft ist in „Familien“ organisiert. Jede Familie repräsentiert ein Element der Zivilgesellschaft wie „Medien“, „Gewerkschaften“ und „NRO“, deren Anzahl von den ursprünglich vorgeschlagenen zehn Familien am Ende der PrepCom 2 auf zweiundzwanzig Familien angestiegen war. Das Büro der Zivilgesellschaft konzentriert sich auf die praktischen Modalitäten für die Beteiligung der Zivilgesellschaft und erstattet grundsätzlich den Plenarversammlungen der Zivilgesellschaft Bericht. Diese tagen nur im Rahmen der PrepComs und des Gipfels; in den langen Perioden zwischen den einzelnen Sessionen ist seine Tätigkeit weniger straff organisiert und es dürfte einem gelockerten Plenum der Zivilgesellschaft Bericht erstatten.

Das Konzept ist in verschiedener Hinsicht problematisch und kann nur schwer als wirklich innovativ beurteilt werden.

Ernsthafte Bedenken gelten der Legitimität und der Funktionsfähigkeit der „Familien“. Die weitgehend willkürliche Aufspaltung der Zivilgesellschaft in 
zweiundzwanzig Teile lässt viele Akteure im Unklaren darüber, welcher (oder wie vielen) sie angehören; die Grenzen zwischen den Familien sind tatsächlich verschwommen, womit die Auswahl der „Vertreter“ nahezu bedeutungslos wird. Die Mitglieder des Büros besitzen zwar bestimmte Befugnisse, aber keine durchsetzbare oder streng kontrollierte Verantwortung bzw. Rechenschaft in Bezug auf den Kreis, die sie vertreten. Viele Familien - wie die „Medien“, „kreative Kreise und aktive Kulturförderer" sowie „Städte und lokale Behörden“" - stehen in einem äusserst diffusen Verhältnis zu den Akteuren, die sie vertreten, und zu ihren Gesprächspartnern in der Zivilgesellschaft. Ausserdem sind weiterhin Verbände des Privatsektors im Büro und in den Familien tätig obwohl das in den Gründungsunterlagen des Büros ausdrücklich untersagt wird (der Grundsatz könnte durch ein angemessenes Selektionsverfahren innerhalb der Organisation problemlos durchgesetzt werden). Derartige Schwächen und Unsicherheiten machen das Büro anfällig für eine ,institutionelle Vereinnahmung" durch die Abteilung Zivilgesellschaft des Sekretariats, die (beabsichtigt oder unbeabsichtigt) ihre Visionen und Prioritäten durchsetzen kann.

Ausserdem werden die weithin anerkannten Hindernisse einer sinnvollen Beteiligung, mit welchen die NRO und die Zivilgesellschaft im Süden konfrontiert sind, höchstens symbolisch - in Form von Reise- und Unterhaltsspesen für eine begrenzte Teilnehmerzahl - anerkannt. Es gelang schlicht nicht, Ressourcen zu finden, um effektive Tätigkeiten auf regionaler und nationaler Ebene durchzuführen. Das Scheitern der Regierungen und anderer Stellen, diese Ressourcen zu mobilisieren, ist an sich sehr vielsagend.

Schliesslich sind weder das Konzept der „Familien“ noch jenes des Büros der Zivilgesellschaft (nicht unbedingt unter der gleichen Bezeichnung) neu. Anlässlich des Erdgipfels von Rio im Jahr 1992 wurde das System der „Major Groups" eingeführt, das sich im Laufe der Jahre zu einem tragfähigen und kohärenten Mechanismus entwickelt hat, obwohl selbst dieser in der Zivilgesellschaft noch Anlass zu Streit gibt. In etlichen Gipfeln wurden Koordinierungsmechanismen wie das Büro zur Erleichterung der internen Koordination und der externen Kommunikation eingesetzt. Die Behauptung bestimmter Kreise, die Gründung des Büros der Zivilgesellschaft schaffe einen innovativen Präzedenzfall, hat sich noch nicht in Form einer offiziellen Anerkennung der Regierungen, neuer Beteiligungsmodalitäten oder Dividenden - bessere Mobilisierung und Organisation - niedergeschlagen.

\section{Zusammengefasst...}

Das oben gezeichnete Bild ist nicht rosig. Die Schuld daran darf jedoch nicht nur der ITU oder dem WSIS zugeschoben werden.

Das allgemeine Klima hinsichtlich der Erneuerung der Weltgipfel und der Beteiligung der Zivilgesellschaft hat sich seit der erstmaligen Ankündigung des WSIS und der ersten PrepCom wesentlich und in einem von wenigen vermuteten Ausmass gewandelt. Was etliche - und dazu zählt sogar der aktuelle Überblick des amtierenden UNO-Generalsekretärs über das Verhältnis zur Zivilgesellschaft als Umschwung gegen die Zivilgesellschaft beurteilen, zieht Kreise weit über 
den WSIS hinaus. Es herrscht ein starkes Vorgefühl heraufziehender Konflikte in den nächsten Jahren - nicht nur mit den USA, sondern auch in der impliziten oder expliziten Zusammenarbeit mit anderen Regierungen und Teilen des zwischenstaatlichen Systems und in Versuchen, den Einfluss der Zivilgesellschaft zurückzudrängen oder sogar die Autorität der UNO selbst zu unterhöhlen.

Zwar waren die Versprechen für eine neue Art Gipfel zweifellos ernst gemeint, aber die Wirklichkeit ist dahinter zurückgeblieben. Die meisten Hoffnungen, die anlässlich der Treffen von Paris geäussert wurden, haben sich nicht erfüllt.

$\square$ Es wurden wenige bzw. keine neuen Modalitäten für die Beteiligung der Zivilgesellschaft gefunden; die bestehenden Modalitäten wurden nicht optimiert. Die Zukunft hält bestenfalls weitere Unsicherheit bereit.

- Es wurde kein Protokoll veröffentlicht, das die Transparenz des gesamten WSIS-Prozesses - vom Büro bis zu den Akkreditierungsverfahren umreisst und bestätigt.

$\square$ Die Zivilgesellschaft erhielt keine Vertretung im Büro; allerdings könnte die Gründung des Büros der Zivilgesellschaft eine bedeutendere Interaktion noch erleichtern.

$\square$ Es fehlt ein abgestimmter Prozess, um die Interaktion der Zivilgesellschaft und die Beteiligung am Gipfel über das Minimum der unzureichend finanzierten CSD hinaus zu fördern; Ideen wie die „Moderatoren“ für die Zivilgesellschaft wurden wegen mangelnder Ressourcen nicht verwirklicht.

$\checkmark$ Die Finanzierung der Zivilgesellschaft bleibt sporadisch und willkürlich. Ein zweckbestimmter Fonds muss erst geschaffen werden. Mit dem Näherrücken des Gipfels sind allerdings in diesem Punkt Verbesserungen möglich.

Ein Hoffnungsschimmer ist noch zu erkennen, insbesondere vor dem breiteren Horizont des zweiten WSIS-Gipfels im Jahr 2005 in Tunis. Der Prozess hat kleinere Öffnungen ermöglicht, die vielleicht zu einer echten Interaktion oder gar einer kleineren Erneuerung heranreifen könnten. Die meisten Regierungen beweisen viel guten Willen, und die ITU, das Sekretariat sowie der WSIS-Präsident haben den aufrichtigen Wunsch geäussert, die Lage zu verbessern. Diejenigen, die am gesamten Gipfel beinahe verzweifelten, sehen vielleicht in der Schaffung eines neuen Modells für die Beteiligung der Stakeholder einen Trostpreis, der auch einige Risiken wert ist. Aus dieser Errungenschaft sollte die Zivilgesellschaft möglichst viel Kapital schlagen.

Damit werden die Alternativen für die Zivilgesellschaft angedeutet. Eine Modalität, die ungewohnt grosse Öffnung beweist, besteht darin, dass die Zivilgesellschaft komplementäre Veranstaltungen zum Gipfel vom Dezember 2003 organisiert. Trotz der allgegenwärtigen Gefahr, zu zweitklassigen Attraktionen mit dürftigen konkreten Ergebnissen oder zu farbigen Nebenveranstaltungen zur Belebung des sonst tristen Prozesses zu geraten, dürften einige Veranstaltungen vielleicht sogar mit dem Haupt-Gipfel rivalisieren, besonders wenn viele Regierungen für die Teilnahme gewonnen werden können. Diese Veranstaltungen sollten nicht am Rande des Gipfels bleiben, sondern das vom Gipfel erzeugte prozedurale und inhaltliche Vakuum füllen. Wenn das Hauptereignis bei den 
Regierungen auf Unzufriedenheit und Frustration stösst, könnten innovative und substanzielle Prozesse für die Interaktion und das Engagement ausgelöst werden. Nur wegen dieser Aussichten bleiben einige Mitglieder der Zivilgesellschaft aktiv, und viele halten sich bis zum nächsten Gipfel daran fest. Das Ergebnis von Genf - in Sachen Inhalt und Verfahren, innerhalb und ausserhalb des offiziellen Gipfels - wird die Entscheidungen der meisten Akteure der Zivilgesellschaft (sowie der Regierung und des Privatsektors), ob weiterhin ein Grossteil der knappen Ressourcen dem WSIS-Prozess gewidmet werden soll, massgeblich beeinflussen. 